\title{
Design, Deployment and Assessment of a Movie Archive System for Film Studies - A Case Study
}

\author{
Nazlena Mohamad Ali ${ }^{1}$, Alan F. Smeaton ${ }^{2}$, Hyowon Lee ${ }^{3}$, Pat Brereton ${ }^{4}$, \\ Finnian Buckley ${ }^{5}$ \\ ${ }^{1}$ Institute of Visual Informatics (IVI), University Kebangsaan Malaysia, Malaysia \\ ${ }^{2}$ CLARITY: Centre for Sensor Web Technologies, Dublin City University, Ireland \\ ${ }^{3}$ Singapore University of Technology and Design, Singapore \\ ${ }^{4}$ School of Communication, Dublin City University, Ireland \\ ${ }^{5}$ School of Business, Dublin City University, Ireland \\ nazlena@ivi.ukm.my
}

\begin{abstract}
While most $R \& D$ activities in the multimedia field today focus on developing new algorithms and tools to automatically understand, index, manage and present media contents so that they could be used to help reduce people's manual efforts in dealing with large amount of media contents, the issues of linking the developing or developed algorithms and tools to realistic end-user scenarios and applications have largely been relegated to other disciplines such as Human-Computer Interaction (HCI) and Interaction Design. In this paper we present our reflection and interpretation of a multimedia project developing an end-to-end novel application incorporating a state-of-the-art "movie scene detection and classification" technique. Unlike any other technology-oriented projects in the multimedia community, this project attempted to bring usability engineering to the core of our experimental system development, extensively adopting the procedures, tools and methods available in usability engineering throughout all stages of the project-user study, ideation, interaction sketching, system implementation and deployment. Largest amount of effort was put to deployment stage, which involved facilitating the web-based system to 256 students in a film study course during a 3 month-long semester, during which the lecturers from the course provided necessary administration and coordination such as access to students, assignments and lab tutorials in order to fully integrate the system to the delivery of the course. Qualitative and quantitative data on the use of the system were recorded over time and analysed, to better understand the nature and characteristics of the developed system and its novel features afforded by the multimedia technology. We summarise the findings and the issues arising from trying to adopt user-centred HCI methods and procedures in developing nonconventional multimedia system throughout all stages of its development.
\end{abstract}

\section{KEYWORDS}

interactive multimedia system, Human-Computer Interaction, usability engineering, film studies, case study, experimental system deployment

\section{INTRODUCTION}

Developing a usable system is crucial because most of user requirement need to fulfill in order to carried out user tasks while interacting with the system. There are number of approaches can be applied to ensure system were developed based on user needs and requirement. Usability Engineering (UE) is one of the approaches that can be used. UE has been useful to support developing usable systems with its rigorous and iterative processes with user interaction and feedback. Many approaches available can be utilized to investigate the user interactions such as observations, think-aloud protocol and ethnographic studies. UE has been always said to design a product with user in mind and very much focus on product.

DOI : 10.5121/ijma.2012.4501 
The International Journal of Multimedia \& Its Applications (IJMA) Vol.4, No.5, October 2012

On the other side, multimedia technology labs have been developing potentially very useful tools, techniques, methods and algorithms, examples such as scene detection, concept detection and object tracking for video. There has been much work carried out regarding the technical possibilities for video content analysis. This dimension of work normally processes video elements with various automatic outcomes and analyses such as shot boundary detection, image segmentation and automatic detection of semantic concepts [1, 2, 3]. Much of this work has as its goal, a focus on the evaluation and measurement of precision and recall, of the efficiency and accuracy of the techniques developed. User involvement, if any, will normally be involved only at the testing stage. Such evaluations are performed in pre-defined lab settings or sessions. Various system interfaces to the new technologies can be developed as a showcase in order to carry out the evaluation. However, not much work has been carried out beyond this boundary where new video analysis techniques are deployed and used by real end-users in real settings and with real tasks involved, and the user interactions with the new software applications that incorporate these kinds of technologies being assessed and monitored, such true user interactions are almost never captured or considered.

Between these two extremes, there are very little attempts to take full advantages of those welldeveloped UE methods to design novel applications for multimedia technology. One reason is that we don't know how to start UE when there is no existing user base or precedence of usage. In this paper, we describe a case study in which major UE methods were adopted to all stages of an experimental system development that incorporate state-of-the-art of multimedia technology. The main focus of this case study paper is to demonstrate how we highlight the challenge and issues of going through the full usability engineering process in designing a multimedia system, deploying and then assessing to real users with real tasks in the context of Film Studies. The work involves a technical setting within the domain of computing and an implementation and evaluation of this within a humanities setting.

The paper consists of the following: Section 2 provides an overview of fundamental areas and some explanations on the inter-related fields particularly Usability Engineering process, Multimedia Technologies and some deployment effort that have been carried out. Section 3 describes the stages of the system development and the use of UE ideas and principles. Section 4 briefly explains the results from the user experiments and deployment. Finally Section 5 discuss the findings into several point of view and the conclusion to our case study in the last section.

\section{BACKGROUND WORK}

\section{Usability Engineering Process}

A user interface is the medium of interaction between the user and system. Human Computer Interaction (HCI) is not only about designing interfaces that are easier to use but also involves studying the reasoning behind the building of specific functionality and the long-term effects that systems will have on humans. It is mainly about how to design systems that support interaction and human use between users and systems. It is about knowledge, context and the interaction between human and computer [4]. The usability roles are important to involve directly in the software-development life cycles. Usability engineering [5] are among the effort made within the $\mathrm{HCI}$ fields from the perspective of the development process life cycle.

Nielsen [5], elaborates the stages of the usability engineering lifecycle model which consist of the summary of activities for system design. This is one of the approaches in user-centred design. Usability Engineering provides a set of tools and methods that can be used and applied in developing a usable product, and this has been refined, tested and proven useful in both research and practice. Usability Engineering focuses on direct/indirect end-user input throughout the 
The International Journal of Multimedia \& Its Applications (IJMA) Vol.4, No.5, October 2012

development process and iteratively refining and correcting according to this input so that the final product would more accurately reflect the end-user needs, thus taking a strong User-Centred Design philosophy at its core.

\section{Multimedia Technologies}

A multimedia system usually integrates one or more related technologies to automatically index multimedia contents (text, images, audio and video), so that consequent access to the multimedia information is enabled. In video, the automatic processing techniques used can be put into several categorical types of work including: shot boundary detection; scene classification; face labelling and recognition; novel browsing, searching and visualization of image/video. For a long time, multimedia systems have been a hot topic with huge potential [6] and also an active research area [7]. One of the main challenges in multimedia systems is in managing the information, and in particular searching.

A video element describes a sequences of moving pictures. At a logical level, a video document can be divided into a set of basic components such as: episode (i.e. a group of related scenes), scene (i.e. a set of consecutive shots that has meaningful semantic value), shots (i.e. a set of consecutive frames) and frame (i.e. a single picture of a movie film and no temporal analysis). Smeaton [7] points out that video data can be retrieved using a number of approaches such as by using metadata and browsing by keyframe, text transcripts search, keyframe matching, semantic feature filtering, object matching and combinations of these techniques. There is a complexity in video elements which need proper organization as compared to when dealing with only text elements. In a huge video database, necessary indicators on the audio, visual and textual elements will help the video material to be more searchable and browsable [8].

There are work related to video content analysis that focusing on the technical possibilities. Research by Lehane [9], used an approach to detect events in a movie and classified them into three classes based on film grammar which is Dialogue (i.e. contains a conversation among characters of one or more people), Exciting (i.e. contains something exciting for the audience like car chase, fighting) and Montage (i.e. contains strong musical background as in montage, emotional and musical events). In making a movie, a director will follow a certain universal film grammar. As for example, they will use a static camera to give an audience low distraction, relaxed viewing-mode and to give more focus. On the other hand, in creating an exciting feeling for viewers, faster pace editing and high camera movement is used to give high impact and increased stimulation levels. These will create a feeling of excitement to viewers indirectly, while music is used a lot as a medium for creating an emotional response among the viewers.

Another work is from INRIA [10] that concerned on browsing and watching movies in synchronization with the scripts and some metadata. Browsing and navigation links were dynamically generated by the server as the user watched, browsed or queried the movie. Browsing and navigation could be done from a DVD or by using the movie keyframe or thumbnails. To our knowledge, no interactions or evaluation has been made during system development and also no evaluation with real users. Theoretically the system would be beneficial to film studies students for movie analysis but in practice, no user experiment was performed so far.

The Virtual Screening Room (VSR) [11] project is an informative browser for playing a movie with features such as clip searching. The development of VSR was meant to be a 'textbook' that can be used in teaching film which specifically focused on the Editing topic. The database stores 500 movie clips related to the Editing Chapter. To our knowledge also, there is no information on the interactions made with real users during the development and evaluation. 
The International Journal of Multimedia \& Its Applications (IJMA) Vol.4, No.5, October 2012

These projects are very useful and effective in understanding, appreciating and teaching movies especially for students in Film Studies. Most application systems mentioned above provide similar features for browsing and searching in a video system. These systems were mainly initiated from a technical perspective of video content analysis, designed and developed as intermediary interfaces to evaluate the proposed techniques or methods. As far as we are concerned, no interactions with real users has taken place in designing and evaluating these systems as their main aim has been on research on the technical possibilities.

\section{System Deployment}

There are other related work carried out on deployment efforts and ethnographic studies. The F'1schl'ar Digital Video Library System was developed at Dublin City University to support capture, indexing, browsing, searching and summarising of digital video and has been deployed into four separate video content collections for a variety of users and application scenarios [12]. Information provided to users in the system interface are based on the finding and selecting a video program either using text or metadata. Supported interface elements included a keyframe slideshow, a hierarchical keyframe browser, and a timeline browser. F'1schl'ar-News was one of the collections designed to support an archive to the main evening TV news broadcast. It incorporates a number of multimedia and recommendation techniques and was deployed within a University campus for several years, in which the large scale testing and evaluation (performance and usability) has been carried out [13]. Methods used from the video content analysis include shot boundary detection, keyframe extraction, capture of closed captions, and the system allows for text searching, browsing and playback. An extended live usage study has been performed on $\mathrm{F}^{\prime} 1 \mathrm{schl}$ 'ar-News for a month with 16 users using a highly qualitative and ethnographic diary [14]. This study mainly emphasized the understanding of real use, the development of use over time and the use of new technologies in new contexts.

Newsblaster at Columbia University [15] is an experimental system incorporating natural language processing techniques to automatically crawl news websites on a daily basis and summarize and present them to web users. The system has been deployed since 2001 and was developed mainly to demonstrate its summarization robustness and the use of Topic Detection and Tracking (TDT) technology. Researchers on the Newsblaster system had started conducting a large online evaluation to measure the usage and user preferences. The system that is available online has also undergone a number of experiments mainly on summarization efficiency (Precision and Recall).

SportsAnno is a video browsing system that is designed and developed to allow users to make comments and share opinions and ideas on soccer events with other registered users [16]. Users are drawn from members within the research group, and friends and outsiders. SportsAnno was deployed during the soccer World Cup 2006 matches. Users can browse soccer video and at the same time make comments and annotate while reading texts of newspaper reports related to the matches. The system provided an up to date information and context on the current match. Usage data on sports event segmentation are gathered during the deployment stage. From the deployment, a number of usage monitoring and issues were pointed out such as low responses for some specific games comments.

F'ischl'ar [12], Newsblaster [15] and SportsAnno [16] are examples of some of the work in deployment efforts that combine technology into practical system development. These trial efforts show a growing awareness of the importance of user evaluation in a realistic environments. Studies that incorporate the end-user perspectives from the conception of the project, are very rare: most of the technology trials start purely from a technical point of view and only after deployment do they get any form of feedback from real usage and users. 


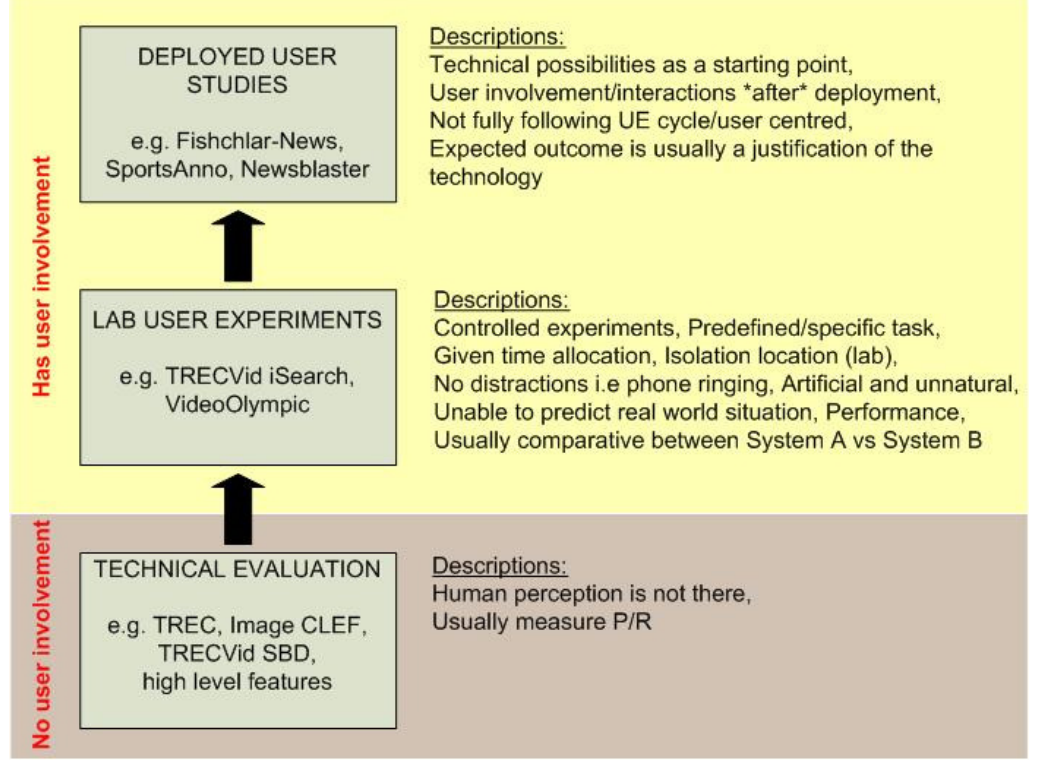

Figure 1: Research perspectives

We provide some arguments in illustrating the research perspective which we believe are important as illustrated in Figure 1. As can be seen in the diagram, there are different stages in a technology development, where technical evaluations were the main concern. Research was mostly carried out to evaluate the effectiveness like measuring the precision and recall of any proposed techniques (bottom part of diagram). Some of the related work includes TREC [17], ImageCLEF [18] and TRECVid SBD [19]. Without the involvement of real users, these overall technologies remain only as technical possibilities. By building an end-to-end system, we can bring in human users into the whole process of development and test the technology in a more natural way. Having users involved in the experiment is normally done by recruiting a number of sample users doing a predefined task in a specific lab or place. Usually the measurements are based on a comparative versions of the system but yet still focusing on the effectiveness of the proposed techniques. In this way, user involvement starts. Examples of research experiments carried out such a way are include numerous interactive search task experiments in TRECVid [20] and VideoOlympics [21], and indeed majority of user experiments in the fields of Multimedia, Information Retrieval and other technologies which try to measure and compare the effectiveness of a proposed technique.

However, working in an artificial lab environment, having time constraints within which to complete searches, working on pre-defined tasks, etc. makes a user experiment artificial and unnatural. With this acknowledgment, a small number of more ambitious studies have started an actual deployment of systems into the real world. All messiness now become part of the evaluation. From the other perspective, these work are not fully user-centered, because their involvement with users can be considered to start AFTER the system deployment happens. In other words, the technology itself was already conceived and developed, the application scenarios constructed, and the interface has been designed, without any end-user involvement.

\section{A CASE STUdy}

This section will briefly explains the stages of the system development and activities applying the usability engineering approaches. The overall summary of our work are represented in a diagram 
The International Journal of Multimedia \& Its Applications (IJMA) Vol.4, No.5, October 2012

in Figure 2. The diagram represents a summary of our interactions with different groups of classes over a 2-year period and the main activities involves throughout the whole work.

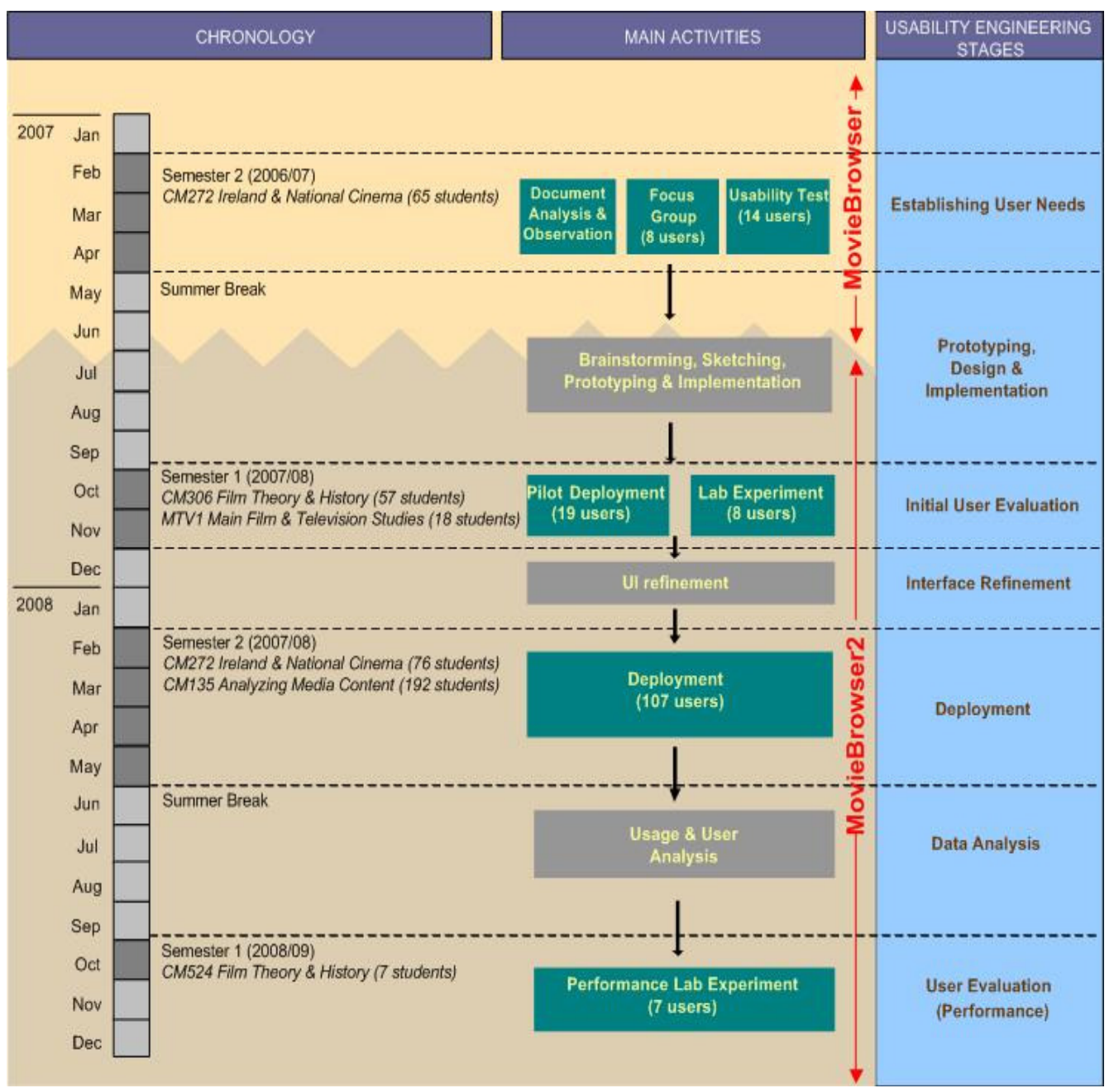

Figure 2: Overview summary

The background to the work was based on work done by Lehane who developed a system called MovieBrowser [9]. The work has an approach to automatic analyse the video content. Lehane's work takes a full movie as a main domain resource, and performs an automatic event segmentation based on scene detection, and automatically classifies each scene into one of three categories (i.e. dialogue, exciting and montage). The main aim in the work is to evaluate and measure the accuracy of the scene detection and to improve its efficiency. In order to do that, Lehane developed a standalone system called MovieBrowser and he performed a series of experiments with users at the final stage of his work. MovieBrowser was not developed with any users or any user tasks specifically in mind from its beginning. In our case study, we take Lehane's work and we incorporate components of the content analysis into the new version of system we called as MOVIEBROWSER2 in which our system does not focus on the accuracy of the video analysis. Our work is not about the development of new video analysis technologies, but on assessing the usage impact and user experiences when using such new technologies. The diagram in Figure 3 shows the overview of a background transition and how it contributes to the case study on this work. 


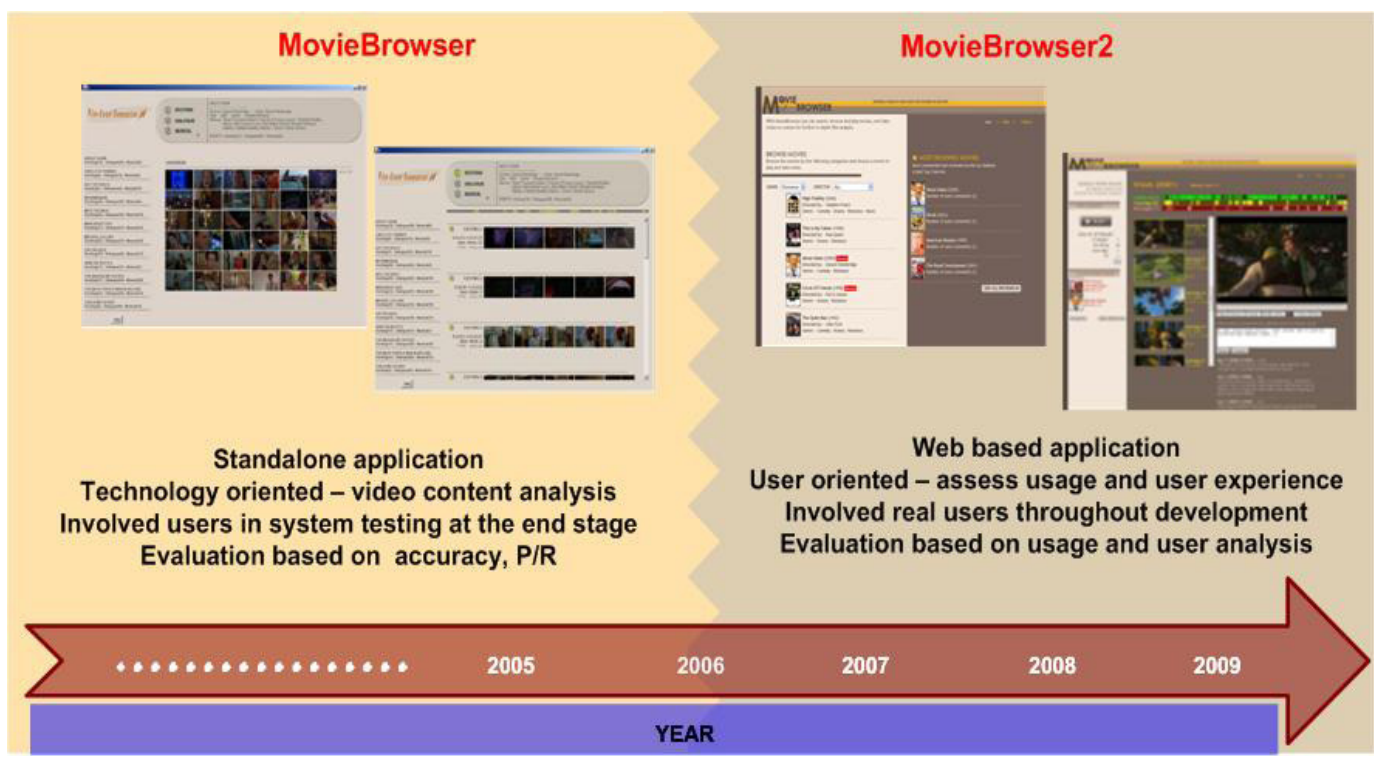

Figure 3 : Background work

\subsection{Requirements gathering and pre-design}

The work started with an interaction with one module of 65 students taking CM272 Ireland and National Cinema course. Our application domain is Film Studies, where students need to study movie contents and to analyse certain movie sequences in order to generate textual essays describing their interpretations of those sequences/clips. The typical tasks of Film Studies students is to read and analyse movies. Reading a movie in their context refers to the process of understanding and analyzing movie content closely, looking for different levels of meaning and critique from elements like framing, depth of field, plot, shots, camera angle, lighting and so on. On a broader level it also involves an understanding of the generic conventions and narrative structure of individual movies [22].

In order to design and developed a new version of system tailored to users need and requirement, we began our work with the identification of user needs through observations, focus groups and usability testing of Lehane's MovieBrowser.

Observations - We carried out observations to understand the way in which students were taught, lectured, and assignments were completed. A consistent observation was made for the whole semester by attending the students' classes where we observed how the whole class was conducted by the lecturer.

Focus Groups - Focus group comprised of an interactive group setting to more actively elicit students' opinions on the course topics as they discussed among themselves, agreed, and disagreed during the sessions. The objective of the focus group was to extract as much direct information from the students on their requirements, needs, and specifications in their film studies. A total of eight participants were recruited, comprising four females and four males, from the Ireland and National Cinema (CM272) module. Six main questions, related to participants' close analysis and their environments when studying the film studies module, were asked during the focus groups. They were asked about the typical approaches that they used in solving problems when working on the tasks given by the lecturer. Questions regarding the resources they used, problems they faced in accessing resources, information needs, and learning goals 
The International Journal of Multimedia \& Its Applications (IJMA) Vol.4, No.5, October 2012

were among the topics of the discussion. The last sections in the focus group were a section called "designing with the users" where we took a brainstorming approach to sketch out a new design based on their requirements. They were asked about what features they would like to have if there were tools that could help in their studies, particularly in analyzing film sequences. The proposed interface designs were sketched on a whiteboard.

Usability Testing - A lab experiment was also carried out with the main objective was to find detailed problems and issues in the initial MovieBrowser system. The main reason for usability testing is to discover usability problems. MovieBrowser was installed in the lab from the beginning of the semester. Early in the course, a system demo was conducted in the class to introduce it to all students. Students were given ample time to familiarize themselves with the system for the first half of the semester before the lab experiment was conducted. In the lab experiment, we had 14 students volunteered. Each experiment took approximately an hour. Participants were given an information sheet and consent was required before they could start the experiment. A set of earphones were provided to each participant. We started the experiment by giving an introduction to the purpose of the experiment, and participants were allowed to ask questions regarding the experiments at anytime during the session.

In order to achieve the objective of the experiment, participants were provided with simple exploratory task. They were allowed to spend 10-15 minutes exploring the system before the timed experiment started. The questionnaires given in the experiment were divided into two parts. Part A was a pretest consisting of five general questions on demographic information including age, gender, available resources for the course, average number of times they watch films and general add-on information for film close-analysis study. Part B was a post test and was a multiple type format. A total of 9 questions were asked which included 5 questions with Likert scales that ranged from 1 to 7 . The questions were focused on system functionality, features provided, interface and overall impression.

\subsection{Design and Implementation}

The MOVIEBROWSER2 took about 9-months of development process. The design and development effort starts from the conceptual design (i.e. interface design layout, sketches and module structuring) and implemented using PHP programming using a streaming technology with Microsoft SQL database. The system has been configured for Microsoft Windows-based (XP), Microsoft Internet Explorer V6+ and VLC player. A screen resolution at least $1024 \mathrm{x} 768$ is recommended. It is also recommended that the machine has $512 \mathrm{MB}$ of RAM and a processor of $2.0 \mathrm{GHz}$ or above.

MOVIEBROWSER2 was designed to support two undergraduates film studies course modules at Dublin City University (CM272 National and Ireland Cinema and CM135 Analyzing Media Content). Libraries of 30 movies were made available on MOVIEBROWSER2. They include various genres (i.e. comedy, drama, romance and action), and ranged from contemporary Hollywood movies to old Irish movies. Details on system design and development were reported in Nazlena et. al [23]. Figure 4 shows an examples of MOVIEBROWSER2 screenshots. Figure 4(a) shows a screenshot of the front page that displays movie posters and some movie information. Filtering by movie genre and director are provided by a drop down list at the top area. Figure 4(b) shows a screenshot of the main viewing area with a visual timeline corresponding to events like dialogue, montage and exciting on the top area, shot keyframe view, the playback area and note-taking section under the playback area. 
The International Journal of Multimedia \& Its Applications (IJMA) Vol.4, No.5, October 2012

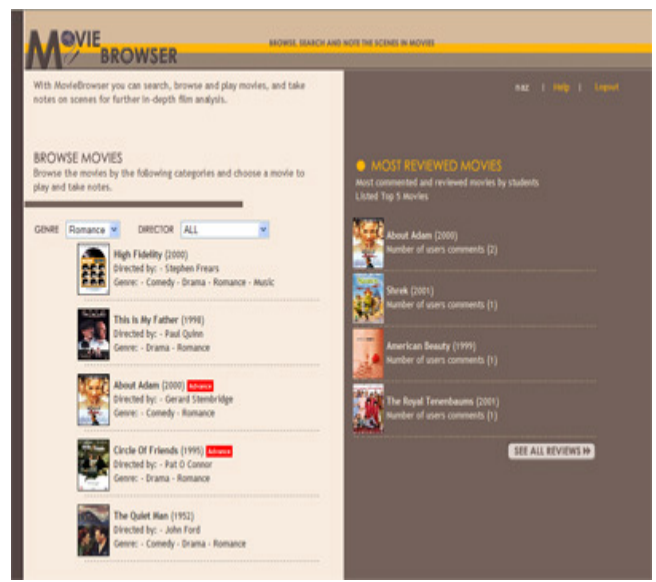

(a)

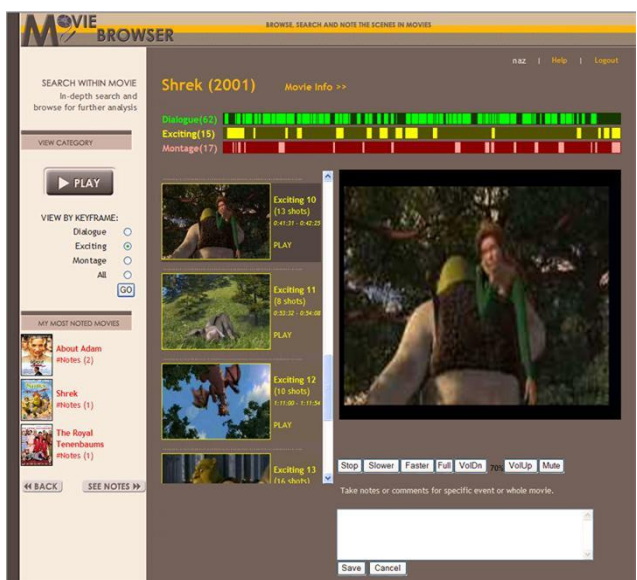

(b)

Figure 4. Interface screenshots: (a): front page, (b) main view area.

The note feature provided in MOVIEBROWSER2 was set to be public, similar to the common commenting features of blog sites. Each shot keyframe represents an automatically detected scene, clicking on a keyframe will play video clips from the scene. The similar colour schemes for event classifications were used in the timeline and the shot keyframe's borderline and text (i.e. yellow colour for exciting events).

\subsection{System Deployment}

Our main goal in this deployment is to study and validate the usage of the technology that we implement in MOVIEBROWSER2, into the real users' tasks as students of Film Studies in the School of Communications, Dublin City University. User feedback and data collection were gathered over a long period of time frame, which corresponding to a whole semester of the University calendar. In the deployment, we are not focusing on the specific features of the interface, but mainly we want to access user interactions among the students in terms of acceptance level.

Participants - Our group of real users at the deployment stage are students from CM272 National and Ireland Cinema which is a second year undergraduate level course of one semester (12 weeks classes) and CM135 Analyzing Media Content, a first year undergraduate level module, also of one semester duration. The combined class groups totalled 268 students (CM272 $=76$ students, CM135 = 192 students). For the former module, the lecturer used mostly an Irish cinema maker as the focus of their main study topic within the module while the latter module used other movies (e.g. contemporary Hollywood).

Tasks-As part of our end-user involvement effort, we deployed our system for the duration of the whole Spring semester. The modules required students to watch or "read" movies and to write essays on specific aspects of those movies, where conventionally the students would borrow DVD movies from the library or use other resources. In providing an additional means to access the movies but much more conveniently than DVD borrowing from the library, we wanted to monitor the detailed usage of the tool throughout the semester in a natural, contextual, and longitudinal way, in which the task was to watch movies, to understand them, and then to write an essay as given by the lecturer of the module. In MOVIEBROWSER2, the movies were separated into two categories according to the type of browsing that was supported; Basic and Advanced. 
The International Journal of Multimedia \& Its Applications (IJMA) Vol.4, No.5, October 2012

The advanced type of browsing will have features that could enhance user browsing, like the inclusion of a visual timeline, support for keyframe browsing, note taking features and movie playback. On the contrary, a movie listed with the standard category of browsing has only a playback function with standard DVD-like player interface facilities such as play, pause, slider bar, etc.

Experimental Procedures - We provided a system demo in the earlier part of the semester for each class. The lecturers for each module were acknowledged and informed of the whole procedure before any deployment was carried out and they gave great support and encouragement for the process to proceed. A brief explanation was given to each class regarding how to use the system during a demo presentation and permission was obtained from the lecturers for this purpose. Each student was assigned a unique username and password for our system. The system could be accessed, starting from week-3 until week-14 in Semester 2 of the academic calendar 2007/2008. Follow-up email remainders were sent a few times during the period of deployment. We administered an online questionnaires in week-13 and week-14 of this initial deployment. Students' usage data logs were captured automatically and saved in our database, similarly to our data collected from online questionnaires.

Data Capture - we separated the captured data from the two main resources as listed below: 1. Usage Logs - Our objective in performing the deployment to the students in the University was to monitor and assess their usage of the developed movie browsing and playback tool. This is done automatically from the system and automatically stored in the database. Among the logs that were captured were the interactions or user actions with the features that we provided on the screen interface.

2. Questionnaires - The objectives of the administered online questionnaires was to measure subjective satisfaction with the new deployed system and to collect quantitative and qualitative data on opinion and feedback on the value of the technology we implemented. It was composed of two parts (A and B). Part A was on the demographic data on students' gender, age, course information and other preliminary inquiries. Part B was on their overall reaction to the system in terms of their perceived satisfaction, views on the features provided, opinions on the value of technology (positive and negative).

\subsection{Focused Lab Experiment}

The analysis of user performance experiment was carried out in the first semester of an academic calendar with a new batch of students of the module Film Studies during Semester 1 of the 2008/09 academic calendar year.

Participants - Our participants for this experiment were from Semester 1 of the 2008/09 academic calendar module which is CM524 Film Theory and History. It is a level 5 Masters class module which is a different group of students from the previous interactions. We recruited all 7 students as there were only 7 students enrolled in this delivery of the module. The aim of module CM524 is to provide students with a theoretical and historical understanding of the development of cinema. One of the learning outcomes of the module is that the student will be able to produce a textual analysis of a wide range of film types. The teaching methods used by the lecturer are by having a lecture and a movie screening each week as part of the same session and this begins with textual analysis exercises. This textual analysis exercise component of the module delivery has been adopted into our study.

Tasks - The task given to students in this experiment is about how to 'read' small sequences of a film. Each student needs to produce a small piece of writing based on reading movie sequences (only writing about parts of the film that are requested in the question) and we will compare how 
The International Journal of Multimedia \& Its Applications (IJMA) Vol.4, No.5, October 2012

students perform this task using MOVIEBROWSER2 versions and using only a standard movie player. Our arguments in choosing this methodology are because we are only concerned with and we only evaluate how participants browse within-movie content from the interface provided to them, either from a standard media player or from the MOVIEBROWSER2 interface, in order to answer the task question put to them. In both tasks, each student needs to produce a small piece of writing as a result of reading movie sequences that we have chosen, which are less than 5 minutes in duration. The sequence should not be longer than that as suggested by the lecturer teaching the module because it will be difficult for participants to read/analyse in a shorter period as in this experiment 30 minutes is the time allocation given for each task. The first task was about reading and analysing the dinner conversation scene in the movie American Beauty (1999), while the second task was regarding comparing and contrasting a few related scenes from the same movie that had musical elements in them. One of the considerations in choosing this as the movie for screening is because it was an award-winning movie which has an interesting plot. Figures 5 show examples of the scene keyframe of the sequences as described in both tasks with the timeframe from within the whole movie. Participants need to browse and analyse relevant scenes or sequences as described in the task by using the assigned interface systems as described below:

Task 1 - Find the scene below (in the movie American Beauty) and perform some analysis of its content. Analyse the techniques used by the director to make the scene more tense between all the family members in the fighting dinner scene where Lester (the father) throws away the food plate onto the wall. [Hint: characters smiling to/at each other in the beginning and there are some recognizable changes in dress etc].

Task 2 - Find the scenes below and perform some analysis. Analyse the director's use of music to express the characters' emotion more cinematically. [Hint: you could contrast these scenes - a scene where Carolyn (the mother) is driving and singing in the car on her way back home and a scene where Lester (the father) and Angela (Jane's friend) are together in the bath].

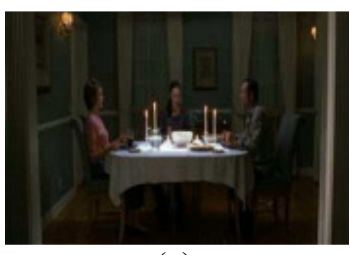

(a)

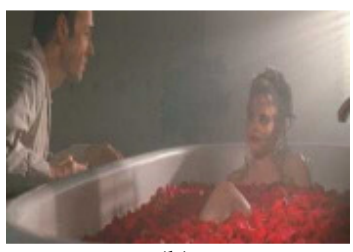

(b)

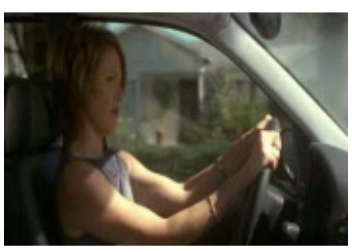

(c)

Figure 5: (a) Task 1 - Keyframe of dinner scene in the movie American Beauty [timeframe 1:05:00-1:07:30], (b) Task 2 - Above: Keyframe of Lester and Angela in a bathtub [timeframe 0:43:35-0:44:12], (c) Keyframe of Carolyn driving a car [timeframe 1:13:45 - 1:14:38]

Experimental Questions - Our main question in this experiment is "Do students who use our newly-introduced technology (in the software tool) get more 'benefit' compared to their conventional way of performing their work task ?". We address this main question by dividing into sub-questions as the following:

Q1. Do students make use of the alternative access features afforded by MOVIEBROWSER 2 and spend less time in completing the essay-writing task?

Q2. Given the same amount of time, is the 'outcome' for those using MOVIEBROWSER2 better or worse than those who use only a standard DVD media player interface for browsing movies? 
The International Journal of Multimedia \& Its Applications (IJMA) Vol.4, No.5, October 2012

Q3. Do the students who use MOVIEBROWSER2 have a higher level of satisfaction than those using only a standard DVD media player?

Experimental Procedures - We separated the experiment into two stages which were carried out in week-3 and week-4 of the academic calendar. In week-3 of the semester, we prepared a slot within participants' regular class schedule. The screening of the movie took about 2-hours carried out in one of the classrooms. The pre-test and consent forms were collected once the screening ended. During week-4, one week after the participants had watched the movie, they were brought to our computer lab in the School of Computing for a controlled lab experiment. This took another 1 hour 30 minutes for this session. There were two tasks that participants needed to perform, and these were assigned in a random order. After each task, participants were given a post-task questionnaire and an exit questionnaire once they finished all tasks.

Participants started with Task 1 first either with the standard media player or with MOVIEBROWSER2. For instance, User 1 must perform Task 1 using interface A (standard movie player) then Task 2 using MOVIEBROWSER2. User 1 can start doing their analysis and write their essay (a maximum of one page) using Microsoft WORD and this process will take approximately 30 minutes maximum. Meanwhile, User 2 began with Task 1 using MOVIEBROWSER2 then followed by Task 2 using the standard movie player. For MOVIEBROWSER2, participants will be given a username and password for their login. A reminder was made 10 minutes before the session ended to individual participants. Once the first task finished, participants needed to submit their Microsoft WORD document as a module assignment through the email address provided. After the email submission, they were given a post-task questionnaire. The same process continued for the following task. When both tasks were finished, an exit questionnaire was then given and the cycle of the experiment ended. There are three CCTV video cameras that captured some of the interactions among participants and used as our secondary support data for our observations. Due to the smaller number of students enrolled in the module, we implemented a within-groups design and to reduce and control the learning effect during the task performance. With this design, participants' experience in Task 1 being used to influence performance to another condition in Task 2 would be reduced.

Data Capture - Data capturing (quantitative and qualitative) for this experiment were measured from various resources as below:

1. Time to complete the tasks - measured from the time the email submission was sent by participants

2. Amount of interactions - measured from observations, video camera footage and screen automatic $\operatorname{logs}$

3. Perceived satisfaction - measured from responses to questionnaires

4. Essays answer quality rating - measured from the essay quality rating either "Very Good", "Good" or "Basic"

Questionnaire design - We administered a pre-task questionnaire in week-3 of the module which is about collecting data on demographics such as age, gender, education background, computer literacy, frequency in watching movies and familiarity with the movie American Beauty. In the following week-4, a post-task was given mainly covering several parts such as one's experiences in performing the task, experiences in using the system/interface and the user's opinions on specific features provided. It is also consisted of open-ended questions for qualitative data collections. Finally in the exit questionnaire, the questions were mainly on the comparison between the two interfaces used in performing both tasks of browsing and analyzing movie contents and the users' experiences and opinions on the experiment as a whole. The role of the 
The International Journal of Multimedia \& Its Applications (IJMA) Vol.4, No.5, October 2012

questionnaire was as an instrument to collect quantitative and qualitative data in answering the research questions.

\section{RESULTS}

This section briefly summarizes the findings from the study.

\section{Stage - User Requirement}

From our observation throughout the semester, students were not really joining in the MOODLE learning system and sharing or exchanging information on movie analysis amongst each other. The discussion in the class tended to be dominated by a few students voicing their ideas. These students will normally start a thread of conversation and continuously lead the sessions.

In the focus groups, most participants talked about how they analysed the scenes in films: overall they look closely at scene-by-scene focusing on, for example, the main characters, dialogue, camera angle, and semiotic means. The second time they watch the scene, they take notes on important points or features and how the scene can influence their feeling. They refer to their notes later when they write essays and reports. One approach to analysing films was to watch a lot of films in the first round to get an overview of possible points and examples available, then focusing on three to four films for closer study. The viewing processes is iterative which means students take notes during the second round of viewing, to see whether the scenes they noted in the first round interest them or not for further comprehension.

Some quotes received from subjects were: "I read all films that I think relevant to get general overview, then analyse those scene in detail, read theories as well", "I pick certain film that acquired certain knowledge that I have, then watch film and break down the analysis, then look at semiotics then go to backup theory then go to online e.g. religion in Ireland". Overall most subjects watched their selected films more than once, by re-playing specific scenes and viewing them and reading their context from the scenes.

Findings from the usability testing on MovieBrowser shows most of our participants used a DVD as their main resources and from the Internet. Many subjects' also complaining on the difficulty in getting the DVDs from the university library. Audio/visual materials such as DVDs have more limited loan period (students mentioned 1-day loan period). For extra information on the films, they referred to Internet resources such as electronic journals, and online free movie databases. Lacks of film material for reference especially for Irish films was among the major problems they had been facing. From the usability experiment on earlier version of MovieBrowser, a list of future enhancements were suggested including a web-based movie browsing and searching system, note-taking features, recommendation search, interactive interfaces, add-on links information (e.g. language glossary, film reviews), and an improved playback features.

\section{Stage - System Deployment}

We found $40 \%$ of the total students used the system during deployment. It is worth noting that the system has a number of constraints such as the access was only from within the University area. A small number of movies were accessed the most, which shows the context of use; for example Irish movies were accessed most due to limited resources from the main University library. From the assessment of system features, we found that students liked and appreciated the afforded features that were incorporated into the tool and affirmed from both the log usage and questionnaires that the tool brings benefit and is helpful to students in performing their tasks. Navigating and browsing the 'Advanced' page shows a pattern of engagement where the time spent was longer than when navigating the 'Basic' page. At first this appeared contradictory since 
The International Journal of Multimedia \& Its Applications (IJMA) Vol.4, No.5, October 2012

the reason for introducing advanced features was to reduce the time taken to perform some task. We relate this observed phenomenon into another perspective which is psychology, in interpreting user engagement with the system as denoting a level of pleasure with having the convenience from the use of the new technology as compared to the conventional approach of going to the University library to borrow a DVD. At the same time, students pointed out their future 'wish list' for improving the tool with larger and more varied type of movie databases, system compatibility with other operating systems besides Windows, improved access from outside the University and some other technical constraints. The mismatches that occurred during the deployment on the features we provided (i.e. note taking) vs. their actual usage, were examined further and design recommendations were made. Usage patterns over time showed that the tool managed to relate to students' real work tasks as heavy usage was seen towards the deadlines for assignments. Students' opinions and experiences in using the software applications and what it meant to them were highlighted. They perceived and appreciated the usefulness of this kind of tool being introduced into their studies. These can be seen from the extracts of their feedback. More results on deployment were reported in Nazlena et. al [24]. Table 1 shows a summary findings and issues arises during the deployment stage.

Table 1: Summary from system deployment

\begin{tabular}{|l|l|l|}
\hline Criteria & Findings & Issues \\
\hline $\begin{array}{l}\text { Movie selection (i.e. Irish and } \\
\text { Hollywood) }\end{array}$ & Positive & $\begin{array}{l}\text { Very limited choices, thus all were fully } \\
\text { accessed }\end{array}$ \\
\hline $\begin{array}{l}\text { Advanced Features (i.e. Timeline visual, } \\
\text { thumbnail playback) }\end{array}$ & Positive & $\begin{array}{l}\text { Being introduced to innovative features as } \\
\text { compared to traditional way of play DVD }\end{array}$ \\
\hline Note taking features & $\begin{array}{l}\text { Negative } \\
\text { (mismatch) }\end{array}$ & $\begin{array}{l}\text { Design should consider the privacy and } \\
\text { conventional methods using pen/notes }\end{array}$ \\
\hline Usage pattern over a semester course & Positive & $\begin{array}{l}\text { Students have no other choices as it was } \\
\text { part of course assignment }\end{array}$ \\
\hline Subjective user acceptance level & Positive & $\begin{array}{l}\text { Hawthorn effect might have occur during } \\
\text { evaluation }\end{array}$ \\
\hline
\end{tabular}

\section{Stage - Analysis of User Performance}

From the experiment we ran after the deployment, we obtained some in-depth information on the benefit students might get from a MOVIEBROWSER2 system such as ours. For Q1, we answered it partially as the findings revealed that users spent time more on the newly introduced tool and they made use of the alternative access features in completing the task through the advanced features provided even though the standard features were available to use. An interesting finding that we found was the time taken to complete the task was longer when completing the same task using the software application, but it produced a slightly better essay outcome. Again, this phenomenon was explained from the perspective of engagement from the psychology domain perspective. The seemingly contradicting fact that the users took longer time with MOVIEBROWSER2 was interpreted from a learning perspective which explains the longer time as proportional to higher level of engagement in using the system as indicated by the student responses to the questionnaire and through researcher observations. For Q2, the student essay assessment in consultation with the lecturer showed that, although the number of tested students, their variability in learning and essay writing skills was an issue, the overall use of MOVIEBROWSER2 resulted in essays showing more various aspects of critical analysis and referring to more example parts of the video, thus receiving better remarks and results as agreed with the lecturer. The findings from the essay outcome revealed that there were slightly an improving or better results which is also supported by the remarks from the module lecturer that shows students have more variability (more opinions, expressions) in the written essay when using MOVIEBROWSER2 that shows an indication of the usefulness of the tool. Q3 was 
The International Journal of Multimedia \& Its Applications (IJMA) Vol.4, No.5, October 2012

answered by looking at the questionnaires after both deployment and the essay experiment as positive. User satisfaction levels were also higher after using the newly-introduced tool with higher means scores in all aspects of statements given as compared to when using a conventional standard player and also from comments. Based on these observations, qualitative 'measurement' and findings, we believe that we have explored and determined that our work (together with deployment experiment) do give impact to users. Further explanation on this experiment were reported in Nazlena et. al [25]. Table 2 listed the summary of the findings and issues related during user experiment stage.

Table 2: Summary from user experiment

\begin{tabular}{|l|l|l|}
\hline Criteria & Findings & Issues \\
\hline Essay writing outcome & $\begin{array}{l}\text { Slightly } \\
\text { improved }\end{array}$ & Control lab experiment which is artificial \\
\hline Time spent & Longer & $\begin{array}{l}\text { Positive user engagement (i.e. flow concept) on } \\
\text { introduced new tool; Confuse interface design }\end{array}$ \\
\hline $\begin{array}{l}\text { Subjective user } \\
\text { satisfaction }\end{array}$ & Positive & Hawthorn effect might have occur during evaluation \\
\hline
\end{tabular}

\section{DISCUSSION}

From a (very) high level of the case study, discussion can be divided as follows:

\section{Applying usability engineering where there is no established user base}

The research approach we took in the work here differed from that had been driven by a technical rationality. The software application was developed emphasizing the usability engineering and user-centred design and was user-driven. The system was deployed into real users (students) and the interaction with real users was our strength over 3 years of experiments with students of Film Studies in Dublin City University. Our aims were to bring a full user-centred approach to enduser interactions, adopt existing video content analysis technology, align the technical rationality of novel multimedia features to a real-world setting, build a usable application when the system incorporates novel multimedia tools that could be potentially useful to the end-users but have not yet been practiced or deployed.

Developing an application when there is no establish user based was actually very costly, because securing the non-existent user base is difficult. We had to create the user base rather than accessing existing users. In our case, a lot of effort required at the early stage of user requirements. We have to carefully choose data collection methods such as observations by involving in the student's class over a semester. This is crucial since we do not know what is user requirement in the course outcome and the expectation from the lecturer and students.

Another issues we foresee in this case study is we already had a technical agenda already in mind when we starts the research which is scene detection and classification of movies. However we see there is a potential in extending the application to be used by 'real' users. In this case students studying Film Studies as these group of users apply lot of film in their syllabus. With these current scenarios, application is no longer experiment in the control lab experiment, but more towards user centered approached. The perspective in the whole process which is user and technical driven and then meets in the middle is difficult. We starts from both directions. We do not have specific guidelines on how we can proceed well in design and development of the application. How do we start from both sides and at the same time and meet in the middle? 
When we conduct user study and asked what they need, we will get those features. This input were then becomes our indicators to be incorporated in the system. But then how do we 'trust' this input? Features that users think important in providing them. For example the notes feature. User said they want in the system, but they didn't use it when it is implemented. Follow up survey shows they needs some privacy in sharing their ideas or knowledge and prefer the conventional way of writing notes using pen and pencil. Thus we could not see these issues while conducting user requirement and was not incorporated in the design.

Long term deployment seems the only way to evaluate, because the novel features provided cannot be lab-tested fairly. In the lab-tested experiment users are mostly seeing the application for the first time, it will not make sense, they will not see the real value of it until they use it for their own purposes. Thus, conducting for long-term, we can monitor how people start using it and identify the value of the feature and start using it. Discovering the value of a novel features can only be done by getting people to start using it and wait for long time. Therefore, a longitudinal type of experiment is useful in this case.

Our work underwent evaluation following the usability engineering cycle with the main focus on the user and applied into a real time frame of a student semester of film studies modules at undergraduates and graduate level. A number of strategies of experimentation used explorative approaches in system deployment and usage monitoring and used predictive approaches in controlled and focused lab experiments, in order to identify user satisfaction, overall performance and system benefit.

In brief, the whole process of usability engineering covers these phases. 1) Establishing user needs where the designer work as close as possible with users particularly in the requirement gathering and getting their insight, 2) Prototyping/design/implementation where the designer follows simplify but rigorously the process of prototyping and design, 3) Initial user evaluation and interface refinement where we follow the UE needs with some iteration in design so that system are user centered, 4) Deployment where this stage is actually a real world problem in going into a real messiness of real usage and most of the issues are identified here, 5) Data analysis where the variability of findings which in certain cases looks failure such as time to engage are longer with new system.

\section{Computing in Humanities}

Bridging between real usage with the work that was driven by technical possibilities and investigate the use of technologies in real usage. Not much work has been carried out beyond the boundaries of technical possibilities from Computing where new video analysis techniques are deployed and used by real end users in real settings and with real tasks involved in the School of Communications, Dublin City University. User interactions with the new software applications that incorporate these kinds of technologies were assessed, where such true user interactions are almost never captured or considered.

Deploying a new technology to be used for real users raise some issues such as user high expectation. From the perspective of real users, students have higher expectation on the technology which they think could help them in completing their tasks without further much thinking on how it works at the back-end engine. They do not borders how is the back end engine works and their aim is only to get the task completed.

Another issues from the deployment that we observe is the usage pattern that reflects positive experiences and outcome from the students. From the perspective of psychology, when someone being introduced to a new technologies they might experience some flow experiences. Flow is theory that reflects engagement in carrying out an activity. Flow theory according to 
The International Journal of Multimedia \& Its Applications (IJMA) Vol.4, No.5, October 2012

Csikszentmihalyi [26] [pg. 4] is "The state in which people are so involved in the activity that nothing else seems to matter; the experience itself is so enjoyable that people will do it even at great cost, for the sheer sake of doing it". Learning environments that are conducive to flow experiences may help people to achieve a positive outcome. Flow is generally reported when someone is doing her/his favourite activity such as cooking a good meal, or gardening and also often happens at work. Any activities can produce flow provided the relevant elements of flow are present making sure that the conditions of flow are present such as clear goals, time flies, skill balanced to action opportunity and the other remaining conditions. Thus it can also occur during this experiment where the features provided in the application might influence them to navigate and browse movie clips better. We observes some positive patterns during deployment and focus user experiment that shows better results and outcome in essay writing.

\section{CONCLUSION}

The work reported here demonstrated our case study in highlighting the issues when applying the UE methods in a development of our application. The case study reflects some emphasize on certain stage in the development process to be fully investigates further in order to reduced user mismatch in design. By building, deploying and assessing an application that incorporates a number of novel multimedia techniques, as we have done in this case study, we demonstrate the application of a user-centred design approach throughout the development of a system and its final evaluation by users in a real, holistic, and contextual manner. Following some procedural way of Usability Engineering is worthwhile and at least the developed artifact already consists of these requirements of being usable and could provide a positive user experience as the results show from our findings. We also learned that following the procedural way consumes time as it involves repetitive cycles but the process itself informed the insight of every stage needed in developing a system which has not yet been used in practice.

\section{ACKNOWLEDGEMENT}

The work was supported by the Ministry of Higher Education and University Kebangsaan Malaysia and by Science Foundation Ireland as part of the CLARITY CSET (07/CE/I1147).

\section{REFERENCES}

[1] A.F. Smeaton. Indexing, Browsing, and Searching of Digital Video. Annual Review of Information Science and Technology, 38:371-410, 2004.

[2] J. Mas and G. Fernandez. Video Shot Boundary Detection Based on Color Histogram. In Proceedings of the TRECVid Workshop, Gaithersburg, Maryland USA, 2003.

[3] A.F. Smeaton, J. Gilvarry, G. Gormley, B. Tobin, S. Marlow, and N. Murphy. An Evaluation of Alternative Techniques for Automatic Detection of Shot Boundaries in Digital Video. In IMVIP'99 3rd Irish Machine Vision and Image Processing Conference, Dublin, Ireland, 1999.

[4] P. Hansen. User Interface Design for IR Interaction: a Task-oriented Approach. In Proceedings of the Third International Conference on Conceptions of Library and Information Science (CoLIS 3): Digital Libraries: Interdisciplinary Concepts, Challenges and Opportunity. Zagreb: University of Zagreb, pages 191-205, 1999.

[5] J. Nielsen. Usability Engineering. Academic Press, Inc., 1993.

[6] B. Furht. Multimedia Systems: an Overview. IEEE Multimedia, 1(1):47-59, 1994.

[7] A.F. Smeaton. Techniques Used and Open Challenges to the Analysis, Indexing and Retrieval of Digital Video. Information Systems, 32(4):545-559, 2007.

[8] A. Haubold, P. Dutta, and J.R. Kender. Evaluation of Video Browser Features and User Interaction with VAST MM. In MM '08: Proceeding of the 16th ACM International Conference on Multimedia, pages 449-458, Vancouver, British Columbia, Canada, 2008. 
The International Journal of Multimedia \& Its Applications (IJMA) Vol.4, No.5, October 2012

[9] B. Lehane and N.E. O’Connor. Movie Indexing via Event Detection. In WIAMIS06 - 7th International Workshop on Image Analysis for Multimedia Interactive Services, Incheon, Korea, 2006.

[10] R. Ronfard. Reading Movies: an Integrated DVD Player for Browsing Movies and Their Scripts. In Proceedings of the 12th Annual ACM International Conference on Multimedia, pages 740-741, 2004.

[11] MIT Center for Educational Computing Initiatives. The Virtual Screening Room. Available from http://caes.mit.edu/projects/virtual screening room/index.htm [Accessed 21 November 2008].

[12] A.F. Smeaton, H. Lee, and K. McDonald. Experiences of Creating Four Video Library Collections with the F'1schl'ar System. International Journal on Digital Libraries, 4(1):42-44, 2004.

[13] A.F. Smeaton, C. Gurrin, H. Lee, K. McDonald, N. Murphy, N.E. O'Connor, D. O'Sullivan, B. Smyth, and D. Wilson. The F'1schl'ar-News-Stories System: Personalised Access to an Archive of TV News. In RIAO 2004 - Coupling Approaches, Coupling Media and Coupling Languages for Information Retrieval, pages 3-17,Avignon, France, 2004.

[14] H. Lee, A.F. Smeaton, N.E. O'Connor, and B. Smyth. User Evaluation of F'1schl'ar-News: An Automatic Broadcast News Delivery System. ACM Transactions on Information Systems (TOIS), 24(2):145-189, 2006.

[15] K.R. McKeown, R. Barzilay, D. Evans, V. Hatzivassiloglou, J.L. Klavans, A. Nenkova, C. Sable, B. Schiffman, and S. Sigelman. Tracking and Summarizing News on a Daily Basis with Columbia's Newsblaster. In Proceedings of the Second International Conference on Human Language Technology Research, pages 280-285, 2002.

[16] J. Lanagan and A.F. Smeaton. SportsAnno: What Do You Think? In RIAO 2007 - Large-Scale Semantic Access to Content (Text, Image, Video and Sound), Pittsburgh, PA, USA, 2007.

[17] E.M. Voorhees. Overview of TREC 2005. In Proceedings of the Text Retrieval Conference (TREC). Gaithersburg, Maryland USA, 2005.

[18] T. Arni, P. Clough, M. Sanderson, and M. Grubinger. Overview of the ImageCLEFphoto 2008 Photographic Retrieval Task. In Working Notes for the CLEF 2008 Workshop, 2008.

[19] P. Over, G. Awad, W. Kraaij, and A.F. Smeaton. TRECVID 2007 - Overview. In Proceedings of the TRECVID 2007 Workshop, Gaithersburg, Maryland USA, 2007.

[20] P. Wilkins, D. Byrne, G.J.F. Jones, H. Lee, G. Keenan, K. McGuinness, N.E. O'Connor, N. O'Hare, A.F. Smeaton, T. Adamek, et al. K-Space at TRECVid 2008. In Proceedings of the TRECVID Workshop, Gaithersburg, Maryland USA, 2008.

[21] P.Wilkins, A.F. Smeaton, N.E. O'Connor, and D. Byrne. K-Space Interactive Search. In CIVR '08: Proceedings of the 2008 International Conference on Content-based Image and Video Retrieval, pages 555-556, Niagara Falls, Canada, 2008.

[22] P. Brereton. How to write textual analysis of a film, CM135 Analyzing Media Content lecture notes. School of Communications, Dublin City University, 2008.

[23] N. M. Ali, A. F. Smeaton, H. Lee (2011), Designing an Interface for a Digital Movie Browsing System in the Film Studies Domain. International Journal of Digital Content Technology and its Application (JDCTA), Vol. 5, No. 9, pp. 361- 370.

[24] N. M. Ali, A. F. Smeaton, H. Lee and P. Brereton (2009), Developing, Deploying and Assessing the Usage of a Movie Archive System. Lecture Notes in Computer Science, In: Human-Computer Interaction. Interacting in Various Application Domains, Volume 5613/2009, pp. 567--576.

[25] N. M. Ali, A. F. Smeaton (2009), Are Visual Informatics Actually Useful in Practice: A Study in a Film Studies Context. Lecture Notes in Computer Science, In: Visual Informatics: Bridging Research and Practice, Volume 58572009, pp. 811--821.

[26] M. Csikszentmihalyi. Flow: The Psychology of Optimal Experience. Harper Perennial New York, 1991. 